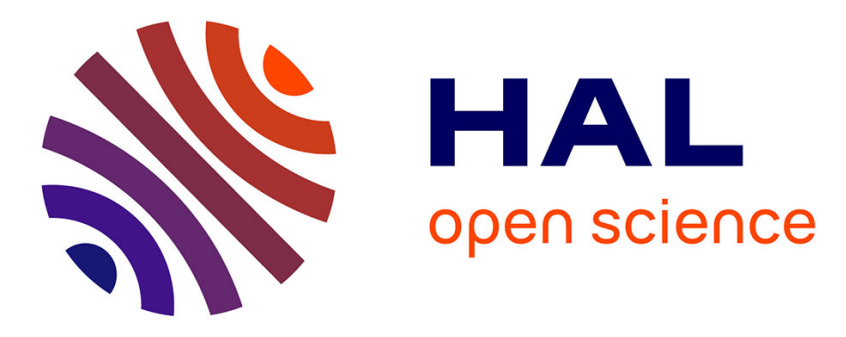

\title{
2D hybrid networks of gold nanoparticles: mechanoresponsive optical humidity sensors
}

Marco Squillaci, Xiaolan Zhong, Léo Peyruchat, Cyriaque Genet, Thomas Ebbesen, Paolo Samorì

\section{- To cite this version:}

Marco Squillaci, Xiaolan Zhong, Léo Peyruchat, Cyriaque Genet, Thomas Ebbesen, et al.. 2D hybrid networks of gold nanoparticles: mechanoresponsive optical humidity sensors. Nanoscale, 2019, 11 (41), pp.19315-19318. 10.1039/C9NR05337A . hal-02413183

\section{HAL Id: hal-02413183 \\ https://hal.science/hal-02413183}

Submitted on 24 Nov 2020

HAL is a multi-disciplinary open access archive for the deposit and dissemination of scientific research documents, whether they are published or not. The documents may come from teaching and research institutions in France or abroad, or from public or private research centers.
L'archive ouverte pluridisciplinaire HAL, est destinée au dépôt et à la diffusion de documents scientifiques de niveau recherche, publiés ou non, émanant des établissements d'enseignement et de recherche français ou étrangers, des laboratoires publics ou privés. 


\section{D Hybrid Networks of Gold Nanoparticles: Mechanoresponsive Optical Humidity Sensors}

Received 00th January 20xx, Accepted 00th January 20xx

\author{
Marco A. Squillacia , Xiaolan Zhong ${ }^{\mathrm{a}, \mathrm{b}}$, Léo Peyruchat ${ }^{\mathrm{a}}$, Cyriaque Genet ${ }^{\mathrm{a}}$, Thomas W. Ebbesen ${ }^{\mathrm{a}}$ and \\ Paolo Samorì*.
}

DOI: $10.1039 / \times 0 \times x 00000 x$

\begin{abstract}
Plasmonic coupling is a fascinating fenomena occurring between neighboring metal nanostructures. We report a straightforward approach to study it macroscopically by fabricating 2D networks of gold nanoparticles, interconnected with hygroscopic organic linkers. By controlling the humidity we tune the interparticle distance to reversibly trigger plasmon coupling collectively over several millimeter.
\end{abstract}

Colorimetric sensors are devices which undergo macroscopic changes in their UV-Vis absorbance or fluorescence characteristics upon interaction with the target analyte. Such changes typically fall within the visible light region and can be so dramatic to be easily perceived by naked eye. ${ }^{1}$ Hitherto, such kind of devices, featuring three-dimensional structures, have been employed for the detection of various compounds in solutions ${ }^{2-4}$ as well as in gaseous state ${ }^{5}$. Noble metal nanoparticles (NPs), which can be synthesized in solution with precise control over shape, size and optical properties610 , represents an extremely versatile platform for the fabrication of such devices. ${ }^{11}$

In solution, gold nanoparticles (AuNPs) are one among the most exploited and best performing scaffolds for sensing because of their high chemical stability, which is achieved upon functionalization with various functional stabilizing shells ${ }^{12}$, and their optical properties, which are extremely sensitive to changes in the local environment. Isolated AuNPs exhibit distinct optical properties arising from their localized surface plasmon resonance (LSPR), ${ }^{13}$ which depends on their size and shape, as well as on the refractive index of their environment. However, when the AuNPs are in close proximity between each other (i.e. with spacing of a few $\mathrm{nm}$ ), a coupling of the LSPR takes place, dramatically modifying the optical properties and therefore altering their macroscopic colour ${ }^{14-18}$. Hitherto, this phenomena has just been exploited for the detection of analytes in solution, where the AuNPs are dispersed or free to move and aggregate, ${ }^{19}$ while sensing of gaseous compounds with dry NPs films is usually achieved through modifications of the refractive index in close proximity to the particle's surface ${ }^{20}$. The latter approach suffers from a poor sensitivity and can only be employed for sensing analytes which exhibit a very different refractive index compared to the one

\footnotetext{
a. University of Strasbourg, CNRS, ISIS UMR 7006, 8 Allée Gaspard Monge, F-67000 Strasbourg, France.

b. Beihang Univ, Dept Phys, Electrochrom Ctr, Beijing 100191, Peoples R China.

Electronic Supplementary Information (ESI) available: [details of any supplementary information available should be included here]. See DOI: 10.1039/x0xx00000x
}

of the shell used for AuNPs stabilization. An interesting strategy to enhance the sensitivity in solid films could rely in the fabrication of dynamic structures in which the interaction with the target analyte affects, at the same time, refractive index and interparticles distance. However, the fabrication of stable structures, providing a predictable and reproducible optical output, allowing to study and to disentangle the two contributions, still remain elusive. In contrast with previous works reporting on unstable superlattices relying on evanescent noncovalent interactions, ${ }^{21}$ here we report on an unprecedented strategy for the fabrication of humidity sensors based on 2D allcovalent networks of AuNPs connected by di-thiolated PEG molecules. The latter acting as a receptor for atmospheric moisture. The peculiar nature of such system allows water molecules not only to modify the local refractive index of the systems, but also to undergo reversible absorption/desorption within the PEG backbone, determining an expansion/contraction of the latter macromolecule and dynamically modifying the interparticle separation, thus their LSPR coupling. The dynamic water physical absorption-desorption and the use di-thiolated linkers enables, for the first time, a fully reversible sensing process featuring response speed of $200 \mathrm{~ms}$ and stability over hundreds of cycles. The displayed shift of the LSPR band is as large as $35 \mathrm{~nm}$ in the visible range (from dry to complete immersion in water) with an unambiguous exponential trend. The mechanically robust $2 \mathrm{D}$ structure of such films should also enable the fabrication of flexible devices.

Water-dispersible AuNPs were synthesized by using an optimized version of the Turkevich method ${ }^{22}$. The conditions for the synthesis were finely controlled yielding particles with an average diameter of $8.6 \pm 1.4 \mathrm{~nm}$, as quantified by Dynamic Light Scattering (DLS) (ESI, Fig. S2). The cross-link of the AuNPs into 2D networks was performed directly in the liquid phase where the AuNPs are grown, without any further purification or separation, by simple addition of water solutions containing controlled amounts of commercial SH-PEG-SH $\left(M_{n}=1000 \mathrm{Da}\right)$. Upon addition of the latter ligand, the solution retains its red colour, endowed by the localized plasmon resonance of AuNPs, yet the characteristic LSPR band undergoes a subtle red shift from 519 to $522 \mathrm{~nm}$ (ESI, Fig. S3). Such shift is due to the different refractive index in close proximity to the particle's surface, providing indirect evidence for the successful ligand exchange from sodium citrate to di-thiolated PEG ${ }^{23}, 24$. Drop-casting the solutions containing the AuNPs/PEG networks onto hydrophilic substrates such as quartz, glass or silicon (freshly ozone cleaned) results in large area monolayers of AuNPs 2D-domains exhibiting high degree of order and tight hexagonal packing, as shown in the Scanning Electron Microscopy (SEM) image in Fig.1. 


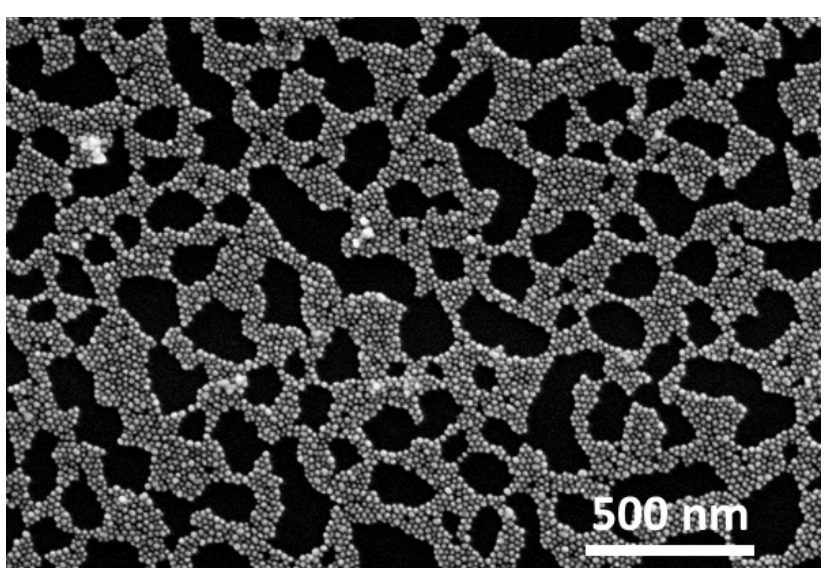

Figure 1: SEM image of drop-cast AuNPS/SH-PEG-SH dry film on Si substrate covered by a native $\mathrm{SiO}_{2}$ layer. The slow evaporation of the water solvent, during the drop-cast deposition of AuNPs/PEG solutions, results in a monolayer of ordered 2D domains of cross-linked AuNPs exhibiting a tightly packed hexagonal lattice.

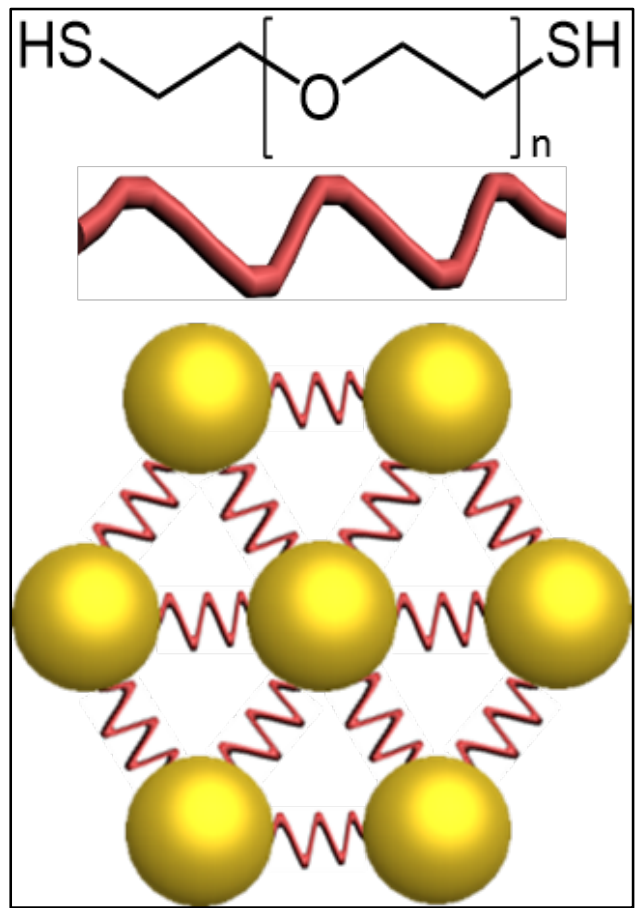

Figure 2: Cross-link reaction of AuNPs with di-thiolated PEG into 2D networks. The use of stoichiometric ratio between the reactive sites on the surfaces of each nanoparticle in solution and the amount of di-thiolated PEG employed for their functionalization, results in ordered and tight hexagonal lattices in which the interparticle distance is defined by the contour length of the employed linker.

The AuNP based 2D networks films display different optical properties compared to isolated AuNPs, as evidenced by a broad SPR band at $595 \mathrm{~nm}$ which can be ascribed to the collective plasmonic resonance of the $2 D$ assembly of closely packed particles ${ }^{18,25}$. In this regime, electrical conductance is due to electronic tunneling between the AuNPs. This can be confirmed by performing twoterminals electrical measurements using electrodes featuring a channel length which is small enough to be bridged by a single domain of packed AuNPs (ESI, Fig. S4 and S5). In our case we employed $100 \mathrm{~nm}$ wide channels and recorded an average resistance of $772 \mathrm{M} \Omega$, indicative of very short percolative paths for the charge carriers, due to the insulating nature of PEG chains and to the large interparticle distances within the network. Such result confirms that the PEG chains are acting as spacers, preventing any interparticle contact and yielding the hcp lattice schematized in Fig. $\mathbf{2}$.

PEG chains have been chosen as bridges between the AuNPs since they are highly flexible and hygroscopic ${ }^{26,27}$. As a result, upon increasing humidity in the atmosphere, the PEG network swells, thereby increasing the interparticle distance. Such swelling has a dramatic influence on both the electrical and optical properties of the film. From the electrical standpoint it causes an important drop of the measured current (ESI, Fig. S6) whereas, from the optical viewpoint, it determines a strong blue shift and a sharpening of the plasmonic band, as shown in Fig. 3. When fully elongated, the employed ligand adopts a nominal contour length of ca. $8 \mathrm{~nm}$, expanding the interparticle distance and reducing the intensity of the interparticle SPR coupling 25 . The effect of humidity causes an overall shift of the SPR band as large as $35 \mathrm{~nm}$, corresponding to a macroscopic color change from blue to red, within the range between $50 \%$ and $100 \%$ Relative Humidity (RH).

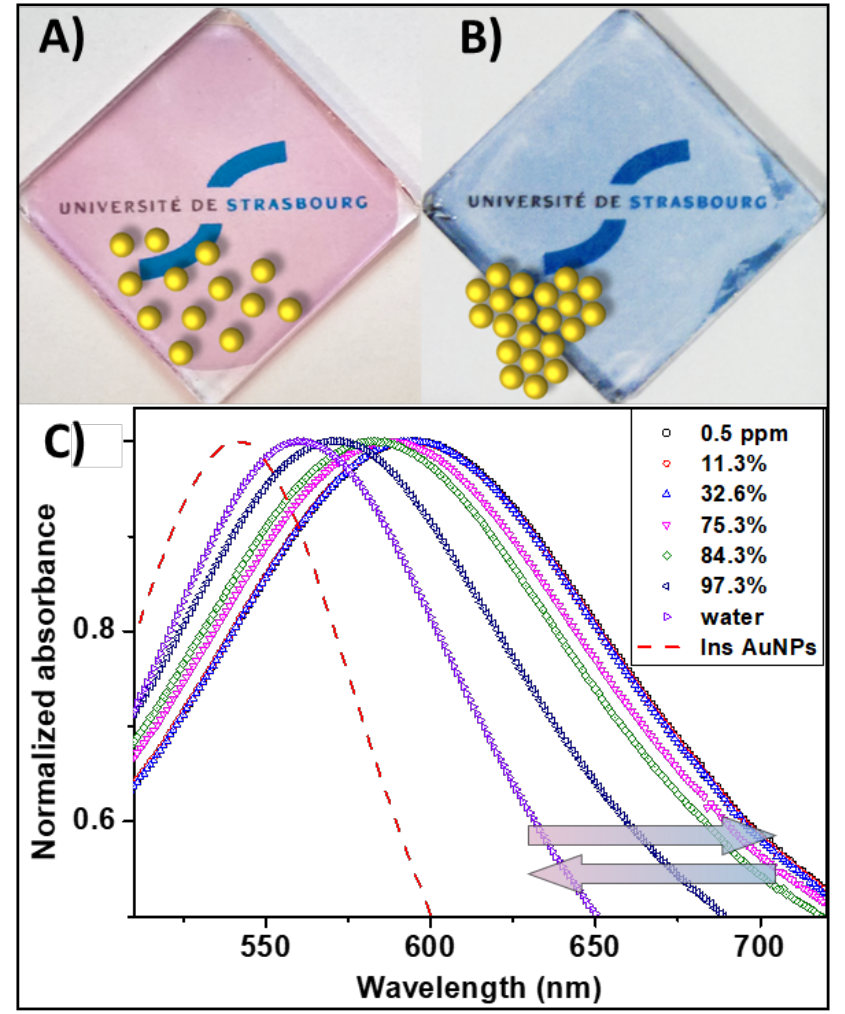

Figure 3: Effect of humidity on the macroscopic optical properties of 2D AuNPs/PEG networks. The increased environmental humidity causes a strong and reversible change of film colour from blue (B) towards red (A). This colour change is given by a shift of the UV-vis absorbance band which moves gradually from 595 to $560 \mathrm{~nm}$, towards a state of decoupled AuNPs (C).

The limit of the blue shift would correspond to a monolayer thick film where all the particles are mostly isolated and electronically disconnected between each other, exhibiting a sharp localized SPR band at $\lambda=535 \mathrm{~nm}$. The optical properties of the uncoupled nanoparticles are reported as a red dashed line in Fig. 3C, which was recorded on a monolayer of the same AuNPs randomly arranged and disconnected between each other, featuring an interparticle distance of $35 \pm 6 \mathrm{~nm}$, as displayed in ESI, Fig. S11. Such moisture absorption/desorption processes and therefore the shift of the SPR band have been found to be extremely fast, featuring a response speed of ca. $200 \mathrm{~ms}$, given by the non-bulky 2D nature of the devices, 
in which the response speed is not limited by the slow diffusion of the target molecules. Moreover, it is fully reversible, with a quick and complete recovery of the initial optical properties, being reproducible over undefined amount of cycles, as shown in Fig. 4A and ESI, Fig. S7.

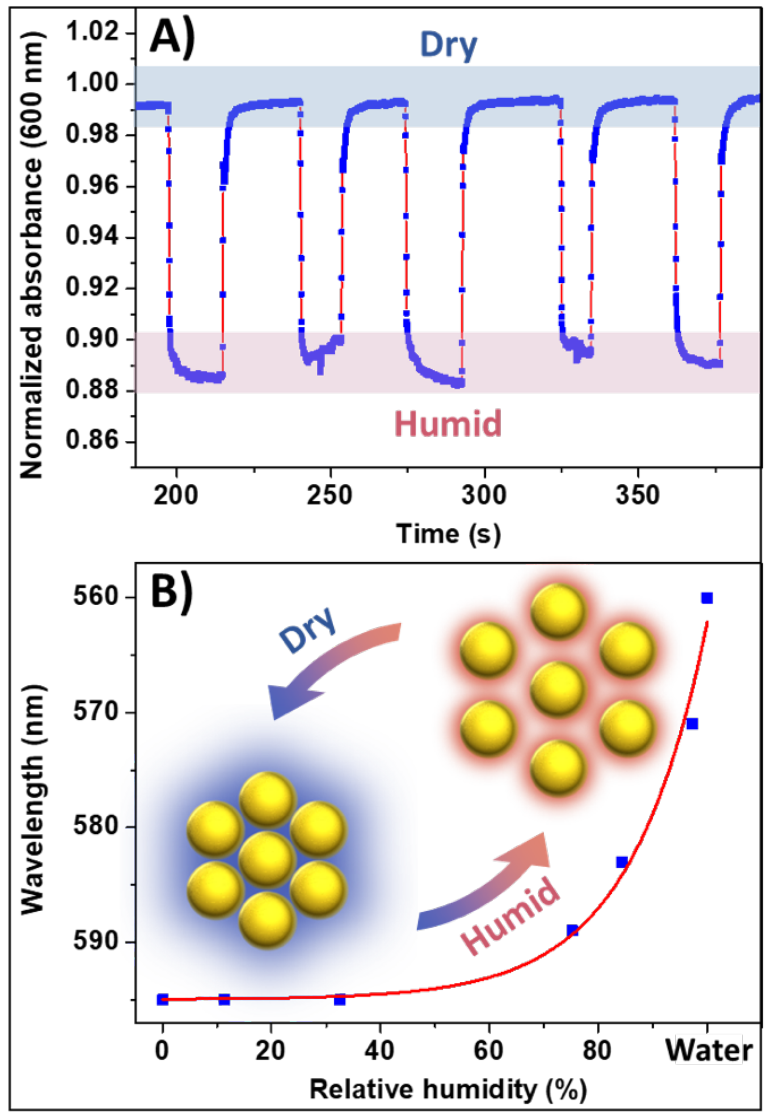

Figure 4: Reversibility of the sensing process. Increased environmental humidity from dry $(\mathrm{RH} \sim 32 \%)$ to humid ( $\mathrm{RH} \sim 75 \%$ ) results in a sharp, fast and fully reversible blue shift in the SPR peak. By monitoring the UV-Vis absorbance at a fixed wavelength of $600 \mathrm{~nm}$ over time, while sending pulses of humid air on the sample's surface it is possible to record the blue shift as a decrease of the normalized extintion measured at that wavelength (A). On the other hand, by recording the whole spectra at different $\mathrm{RH}$ it is possible to plot the evolution of SPR band upon humidity changes, finding a precise exponential dependency (B).

The use of di-thiolated ligands and cross-linked networks guarantees a perfect programmability over the optical properties by using humidity as remote control of the interparticle distance, without modifying the lattice geometry. This aspect is essential to tune the optical properties with an outstanding precision in absence of hysteresis effects. Noteworthy, the shift of the SPR band versus the relative humidity follows an exponential evolution in the whole range of humidity not only from $0 \%$ up to $97 \%$ but also upon complete immersion of the films in water, as displayed in Fig. 4B. These unusual optical properties of the network can be ascribed by the effect of the modification of the refractive index surrounding the particles, due to the absorption of water molecules, which is amplified by the increase of the average distance between the particles as the PEG matrix swells. The low response regime found at $\mathrm{RH}<50 \%$ is due to the modest water uptake within the hygroscopic PEG linkers and can be improved by functionalizing the linkers with more hygroscopic side chains or by embedding a highly hygroscopic salt, such as $\mathrm{LiCl}$, inside the PEG matrix. ${ }^{28}$

To gain further insight into the specificity of our sensor, we have performed discrete dipole approximation (DDA) simulations on hexagonal AuNP lattices of $9 \mathrm{~nm}$ diameter large particles. In "humid" $(\mathrm{RH}=94 \%)$ conditions, the experimental absorbance of the AuNP/PEG network can be well fitted assuming a separation distance between the AuNP of $\sim 8 \mathrm{~nm}$, coherently with the nominal chain length of the fully elongated molecular linker. Such non-contact conditions imposed by the PEG chains lead us to fix an effective refractive index of $\mathrm{n}_{\mathrm{eff}} \sim 1.54$.

Then, we determine the change in $\mathrm{n}_{\mathrm{eff}}$ induced by the desorption of water upon changes in the environmental humidity from the experimental data on PEG water loss/uptake found in literature ${ }^{28}$. This leads us to fix the background refractive index of the network to $\mathrm{n}_{\text {Back }} \sim 1.78$, a value that is greater than the tabulated refractive index of PEG ( $n_{P E G} \sim 1.45$ ). This might seem surprising but, as reported in the literature ${ }^{29}$, such hybrid AuNP-polymer medium behaves as a metamaterial which can be have a large background refractive index. Our simulations provide further evidence that such a large shift in the experimental SPR extinction results from the intertwined contribution of the background refractive index and of the progressive increase of the inter-particle distances as humidity increases, as clearly seen in ESI, Fig. S9 and $\mathbf{S 1 0}$. We evaluated an overall change of the interparticle distance of almost $4 \mathrm{~nm}$, between dry $(\mathrm{RH}=11 \%)$ and wet $(\mathrm{RH}=94 \%)$ conditions.

\section{Conclusions}

In summary, we have demonstrated that the engineering of 2D all-covalent networks of AuNPs enables the fabrication of humidity responsive plasmonic nanostructures exhibiting a large shift of the SPR band in the visible range which can be detected by naked eyes. The remarkable stability over an undefined amount of cycles combined with an excellent response speed of $200 \mathrm{~ms}$ and full recovery makes these architectures appealing for portable and on-skin chemical detection of different analytes when the ad-hoc receptor is employed. Our versatile approach, relying on the synthesis and processing carried out in water, is suitable for deposition of centimetre-scale uniform films onto virtually any kind of hydrophilic substrate for emerging applications in nanomedicine and environmental monitoring. However, more detailed studies will have to be performed to evaluate the mechanical properties of AuNPs hybrid 2D networks after multiple bending cycles for integration on artificial skins. Our tuneable plasmonic structures can also have impact in other field of technology, such as optical-based fast communications, where plasmonic antennae are expected to play a key role in the next future.

\section{Conflicts of interest}

There are no conflicts to declare.

\section{Notes and references}

This work was financially supported by EC through the ERC project SUPRAFUNCTION (GA-257305) and the Marie Sklodowska Curie ETN projects SYNCHRONICS (GA-643238) and iSwitch (GA642196), the ANR Equipex Union (ANR-10-EQPX-52-01), the Labex 
projects CSC (ANR-10LABX-0026 CSC) and NIE (ANR-11-LABX0058 NIE) within the Investissement d'Avenir program ANR-10IDEX-0002-02, and the International Center for Frontier Research in Chemistry (icFRC).

1. Y. H. Christy, M. Liu, Y. Li and J. D. Brennan, Angew Chem Int Ed, 2018, 57, 4549-4553.

2. R. Elghanian, J. J. Storhoff, R. C. Mucic, R. L. Letsinger and C. A. Mirkin, Science, 1997, 277, 1078-1081.

3. C. Zhang and K. S. Suslick, J Am Chem Soc, 2005, 127, 11548-11549.

4. J. H. Holtz and S. A. Asher, Nature, 1997, 389, 829-832.

5. N. A. Rakow and K. S. Suslick, Nature, 2000, 406, 710-713.

6. M. Grzelczak, J. Perez-Juste, P. Mulvaney and L. M. LizMarzan, Chemical Society reviews, 2008, 37, 1783-1791.

$7 . \quad$ L. M. Liz-Marzan, Langmuir, 2006, 22, 32-41.

8. O. M. Bakr, V. Amendola, C. M. Aikens, W. Wenseleers, R. Li, L. Dal Negro, G. C. Schatz and F. Stellacci, Angew Chem Int $E d, 2009,48,5921-5926$.

9. A. Sanchez-Iglesias, I. Pastoriza-Santos, J. Perez-Juste, B. Rodriguez-Gonzalez, F. J. G. de Abajo and L. M. LizMarzan, Adv Mater, 2006, 18, 2529.

10. O. M. Bakr, B. H. Wunsch and F. Stellacci, Chem. Mat., 2006, 18, 3297-3301.

11. W. D. M., S. M. L., N. G. V., G. E. Aitzol, L. Mark, S. Alberto and D. J. A., Adv. Mater., 2018, 30, 1703912.

12. J. C. Love, L. A. Estroff, J. K. Kriebel, R. G. Nuzzo and G. M. Whitesides, Chem Rev, 2005, 105, 1103-1169.

13. L. Guo, Y. Xu, A. R. Ferhan, G. Chen and D. H. Kim, J Am Chem Soc, 2013, 135, 12338-12345.

14. Z. Nie, A. Petukhova and E. Kumacheva, Nat Nanotechnol, 2010, 5, 15-25.

15. C. Hamon, S. Novikov, L. Scarabelli, L. Basabe-Desmonts and L. M. Liz-Marzan, ACS Nano, 2014, 8, 10694-10703.

16. C. Hamon and L. M. Liz-Marzan, Chem. Eur. J., 2015, 21, 9956-9963.

17. A. Klinkova, R. M. Choueiri and E. Kumacheva, Chemical Society reviews, 2014, 43, 3976-3991.

18. A. Tao, P. Sinsermsuksakul and P. Yang, Nat Nanotechnol, 2007, 2, 435-440.

19. E. Priyadarshini and N. Pradhan, Sensor Actuat B-Chem, 2017, 238, 888-902.

20. K. M. Mayer and J. H. Hafner, Chem Rev, 2011, 111, 38283857.

21. C. Kunstmann-Olsen, D. Belic, D. F. Bradley, M. P. Grzelczak and M. Brust, Chem Mater, 2016, 28, 29702980.

22. J. Turkevich, P. C. Stevenson and J. Hillier, J. Phys. Chem., 1953, 57, 670-673.

23. K. Saha, S. S. Agasti, C. Kim, X. Li and V. M. Rotello, Chem Rev, 2012, 112, 2739-2779.

24. L. M. Liz-Marzán, M. Giersig and P. Mulvaney, Langmuir, 1996, 12, 4329-4335.

25. V. Amendola, O. M. Bakr and F. Stellacci, Plasmonics, 2010, 5, 85-97.

26. M. A. Squillaci, L. Ferlauto, Y. Zagranyarski, S. Milita, K. Mullen and P. Samori, Adv. Mater., 2015, 27, 3170-3174.

27. H. M. L. Thijs, C. R. Becer, C. Guerrero-Sanchez, D. Fournier, R. Hoogenboom and U. S. Schubert, J Mater Chem, 2007, 17, 4864-4871.
28. J. A. Baird, R. Olayo-Valles, C. Rinaldi and L. S. Taylor, J Pharm Sci, 2010, 99, 154-168.

29. M. Brust, D. Bethell, C. J. Kiely and D. J. Schiffrin, Langmuir, 1998, 14, 5425-5429. 